ISSN: 2637-9627

\title{
Where do we Stand After PCV13 Vaccine Introduction?
}

\author{
*Corresponding Author(s): Simon Kayemba-Kay's \\ Department of Pediatrics, Centre Hospitalier des Pays \\ de Morlaix, 15, Rue du Kersaint Gilly, 9672 - Morlaix/ \\ France \\ Tel: +33-2-98-62-71-70, Fax: +33-2-98-62-69-86; \\ Mail: kkayembakays@ch-morlaix.fr
}

Received: Jun 24, 2020

Accepted: Jul 28, 2020

Published Online: Aug 03, 2020

Journal: Annals of Pediatrics

Publisher: MedDocs Publishers LLC

Online edition: http://meddocsonline.org/

Copyright: @ Kayemba-Kay S (2020). This Article is distributed under the terms of Creative Commons Attribution 4.0 International License

Keywords: Vaccines; Infections; VBT; VF; Streptococcus pneumoniae.

\section{Introduction}

Pneumococcal infections remain a serious public health challenge across the globe, with high morbidity and mortality. Worldwide, these infections claim over half a million deaths annually in developing countries [1]. S. pneumoniae, a gramnegative bacterium, is a common finding in human nasopharyngeal flora, particularly in toddlers attending daycare facilities in whom, up to $40 \%$ are carriers [2,3]. S. pneumoniae causes both non-invasive (otitis media, sinusitis, pneumonia) and invasive infections such as bacteremia, meningitis, and septicemia in children. It is a complex bacterium that contains over 90 known serotypes present in its polysaccharide capsule, each having different prevalence and multifaceted virulence [4]. The bacterial polysaccharide capsule is important for its virulence, and, it is strongly anti-phagocytic in a non-immune host [5].

\section{Abstract}

Conjugate anti-pneumococcal vaccines are available in routine practice for over two decades now. They have enabled tremendous progress to be achieved in the prevention of severe Streptococcus pneumoniae infections in both children and the elderly. S. pneumoniae is a complex bacterium that has over 90 capsular serotypes each with specific virulence. The advent of PCV13 after PCV7 was expected to further reduce the burden of pneumococcal infections as non-vaccine serotypes started emerging a few years after PCV7 was introduced. Even though much has been achieved and several lives saved, we are faced, in recent years, with cases of Vaccine Break Through (VBT) and Failure (VF).

Through this overview, clinicians are reminded that some vaccine serotypes are less immunogenic, therefore, wellvaccinated children may present with severe S. pneumoniae infections as shown by several reports.

We believe that reporting all cases of VBT and VF is of utmost importance as it would strengthen our epidemiological knowledge and aid in public health policy decisionmaking processes.

In the case of vaccination, however, antibodies are raised against the capsule and are protective only against the specific serotype contained in the vaccine.

The S. pneumoniae capsule is made of several components, of which peptidoglycan and teichoic acid, released upon lysis of the bacterium, act as potent mediators of inflammation in the host. Pneumolysin, another capsular component, is a potent inflammatory mediator that is also released upon lysis of the bacterium. It then stimulates the production of TNF- $\alpha$ and IL- 6 in macrophages and causes lysis of host cells. Pneumolysin plays an important role in hearing loss that occurs following pneumococcal meningitis in about $30 \%$ of affected children [6-9]. Zinc metalloprotease, a protein produced by the bacterium degrades immunoglobulins $\mathrm{A} 1$ and facilitates colonization. Lastly, 
the pneumococcal surface protein antigen is indispensable to the bacterium for it to be fully virulent [9].

\section{Transmission}

S. pneumoniae is spread in person-to-person fashion, through contact with secretions from colonized individuals. Colonization is more common and higher in early childhood, especially in preschool children [10]. In developed countries, toddlers under the age of 2 years constitute the most vulnerable population group as they have a high rate of daycare attendees. This group also has the highest infection rates. Fortunately, the carriage of serotypes with high invasive potential is usually shorter in duration [11]. (IPD)

Risk factors for invasive pneumococcal disease in childhood

Among several known risk factors, age below two years is an important susceptibility factor. Overall, in the general pediatric population (age 0 to 15 years), a predisposing factor for IPD is relatively frequent, with a prevalence of $20-25 \%$. Among these, crowding, presence of older siblings, passive smoking in the household, and the season of the year are those most commonly reported [12-14]. Children with conditions such as those associated with the poor synthesis of immunoglobulins (primary or acquired hypogammaglobulinemia), beta-cell dysfunction, complement deficiencies, and defects in the TLR pathway, congenital or acquired asplenia, HIV infection, lung affections, neurological, and chronic heart diseases are at high risk of contracting IPD [15-20]. It is worth mentioning that any condition associated with CSF leakage (head trauma with a skull fracture and/or inner ear malformation) also constitutes a predisposing risk factor for IPD.

Conjugate pneumococcal vaccination and post-vaccination era

Polysaccharide pneumococcal vaccines (PPV23 and PPV14) have been available since the late 1970s. But, because of their being T-cell independent antigens, they are non-immunogenic in children under the age of 2 years, the ones at the highest risk of developing serious pneumococcal infections. Thanks to advances in medical and pharmacological research, the development of Pneumococcal Conjugate Vaccines (PCV) in which polysaccharides are conjugated to a carrier protein resulting in a T-cell dependent immune response, with the induction of memory cells in very young infants and children, has been possible. Besides, PCV gives an additional possibility for a booster response in case of subsequent polysaccharide antigen exposure. PCVs also have beneficial effects on the nasopharyngeal carriage via the vaccine-induced IgG which may spread to the mucosal lymphoid tissue. The impact of PCV introduction on the global pediatric morbidity is universally acknowledged by several studies [21-25]. But, a few years after the implementation of PCV7, the first-ever conjugated anti-pneumococcal vaccine made available for routine clinical use, concern was expressed over the emergence of infections due to the non-vaccine serotypes $[26,27]$. This, subsequently, led to the development of PCV13.

\section{Where do we stand in the post-PCV13 era?}

Tremendous progress has been accomplished in the field of pneumococcal infection prevention, initially with the advent of PCV7 followed by PCV13, the benefits of which have been universally acclaimed. The immunogenicity studies carried out after the implementation of PCV13 have shown its ability to induce antibody production well above the recommended coverage level $(\geq 0.35 \mathrm{mcg} / \mathrm{ml}$ ) against the six additional serotypes (1, $3,5,6 \mathrm{~A}, 7 \mathrm{~F}$, and 19A) that had caused concern after the PCV7 introduction, in $98-100 \%$ children who had received three injections $[28,29]$. This optimistic response has, since, been disputed by some authors, particularly with serotype 3 , based on clinical experiences with vaccine failures [30,31].

It, therefore, appears that a challenge remains, as cases of vaccine failure are being reported [32-36]. It has also been emphasized that some vaccine serotypes are less immunogenic, and still cause some concern in clinical practice even in wellvaccinated children $[33,37]$.

In an animal model study aimed at evaluating the correlation of pneumococcal capsule size (serotypes 3, 4, 19F and a mutant capsule-free serotype 4) with pulmonary neutrophil extracellular traps (NETs) and disease severity in the mice lungs, Moorthy et al. showed that the serotype 3 (thickest capsule) induced the most severe infection, and was resistant to neutrophil-mediated killing. The serotype 3 also caused the highest extent of pulmonary NETs in comparison to other serotypes studied namely 4, $19 F$, and mutant serotype 4 . This study also highlighted the fact that pneumococcal capsule which is different in each serotype, facilitates evasion from entrapment by NETs [38].

Another particularity with pneumococcal serotype 3 was reported by Trück et al. in a study evaluating the Memory B cell response to a PCV-13 booster in 3.5-year-old children primed either with PCV-7 or PCV-13. These authors found a strong inverse correlation between antibody concentrations and opsonophagocytic activity titers at baseline and B memory cell response for the serotype 3 after vaccination booster, suggesting that pre-existing serotype-specific antibodies likely inhibit B memory formation in response to vaccination [39].

\section{What about SBI and IPD after PCV13?}

It is reasonable wisdom to accept that vaccines do not confer $100 \%$ efficacy against all incorporated antigens, although we would expect that all correctly vaccinated children become entirely protected. Vaccine breakthroughs and failures may occur as a result of incomplete vaccination, incomplete coverage of strains, serotypes, genotypes, antigenic variants, the existence of risk factors, etc. [32].

Vaccine breakthroughs are defined as cases of infection occurring in incompletely vaccinated individuals. Vaccine failure, on the other hand, is defined, according to the Council for the International Organizations of Medical Sciences and the World Health Organization Working Group, as the occurrence of a specific vaccine-preventable disease in a fully vaccinated person, after sufficient time has elapsed ( 2 weeks or longer after the last dose of a given vaccine) for protection against the antigens of the vaccine to develop [40]. In a French study covering the period from 2003 to 2013, Godot et al. reported a rate of vaccine breakthrough (VBT) of $3.2 \%$ (24/943 in PCV7 era, 15/290 in PCV13 era) cases and 0.6\% cases of vaccine failure (VF) (6/943 in PCV7 era and 2/290 in PCV13 era). In this study, both VBT and VF although rare were commoner in toddlers, serotype 19F was the most prevalent even after the introduction of PCV13. It is worth mentioning in this study, that of 15/290 PCV13 vaccine failures, 11 cases of meningitis occurred in children with underlying risk factors [35]. In another study in the USA, Olarte et al., reporting on the im- 
pact of PCV 13 on pneumococcal meningitis in eight US hospitals, concluded that despite the introduction of PCV13 the number of pneumococcal meningitis remained unchanged whereas the proportion of vaccinal serotypes decreased significantly. S. pneumoniae serotype 19A was the most frequent causative agent during their study period $(2011-2013)$. These authors also observed a significant decrease in PCV13 serotypes (from $54 \%$ to $27 \%$ ), non-vaccine serotypes represented $73 \%$ of isolates during the same period [36]. In Sweden, Galanis et al. reported a decrease in IPD incidence following PCV7 introduction, but not with PCV13 in vaccinated children. Following PCV13 introduction, serotypes $6 \mathrm{~A}$ and $19 \mathrm{~A}$ decreased whereas serotype 3 remained unchanged. The vaccination had a real impact on meningitis, septicemia, and severe rhinosinusitis, but not on bacteremic pneumonia in young children [41]. Cases of vaccine breakthrough and failure have also been reported in Catalonia by Fernando Moraga-Llop et al. [42] and more recently by Hernandez et al. [43]. In England and Wales, a substantial reduction in IPD has been observed following the introduction of PCV13; these results are, however, being compromised by the rapid increase in infections caused by non-PCV13 serotypes [44]. In Australia, Zimmermann et al. have reported, in children vaccinated per the local schedule of $3+0$, a level of antibody below the protection threshold at the age of 13 months. Children were, especially, less protected against serotypes 4, 19A, 23F, and $6 \mathrm{~B}$, with the lowest antibody levels recorded for serotypes $4,19 A, 23 F$, and 3 [45]. Cases of vaccine failures have also been reported in children aged $>1$ year since the introduction of $3+0$ vaccination schedules with PCV13 [46-48]. There remains an unanswered question that was put forward as far back as 2014, by Andrews et al. of, whether the defined antibody levels of $\geq 0.35$ $\mu \mathrm{g} / \mathrm{ml}$ for IPD and $\geq 0.5 \mu \mathrm{g} / \mathrm{ml}$ for mucosal disease hold for all PCV13 serotypes. We speculate, like others, that the degree of virulence varying with each serotype; it could, likely be that induced antibody level following vaccination is also variable [49]. More clinical research is needed to clarify this particular point. The precise incidence of both VBT and VF is hard to ascertain, we are, therefore, tempted to believe that both VBT and VF cases are probably under-reported worldwide.

\section{Conclusion}

The advent of PCVs has led to tremendous progress in the prevention of pneumococcal infections with a substantial reduction in both morbidity and mortality in the pediatric population. The emergence of non-vaccine serotypes is, however, a challenge that we are faced with, in addition to several other aspects such as vaccine failure and low immunogenicity of some serotypes contained in PCV13, the serotype 3 in particular. Clinicians must remain vigilant in caring for infected children irrespective of their vaccination status. They are also invited to be pro-active in reporting cases of VBT and $V F$, to enable the global $S$. pneumoniae epidemiological comprehension and surveillance.

\section{References}

1. WHO 2012. Estimated Hib and pneumococcal deaths for children under five years of age, 2008.

2. Syrjänen RK, Kilpi TM, Kaijalainen TH, Herva EE, Takala AK. Nasopharyngeal carriage of Streptococcus pneumoniae in Finnish children younger than 2 years old. J Infect Dis 2001; 184: 45459.

3. Bogaert D, De Groot R, Hermans PW. Streptococcus pneumoniae colonization: the key to pneumococcal disease. Lancet Infect
Dis 2004; 4: 144-154.

4. Kalin M. Pneumococcal serotypes and their clinical relevance. Thorax 1998; 53:159-162

5. Nuorti JP, Butler JC, Farley MM, Harrison LH, McGeer A, et al. Cigarette smoking and invasive pneumococcal disease. Active Bacterial Core Surveillance Team. N Engl J Med. 2000; 342: 681689.

6. Beurg M, Hafidi A, Skinner L, Cowan G, Hondarrague Y, et al. The mechanism of pneumolysin-induced cochlear hair cell death in the rat. J Physiol 2005; 568: 211-227.

7. Tsuprun V, Cureoglu S, Schachern PA, Ferrieri P, Briles DE, et al. Role of pneumococcal proteins in sensorineural hearing loss due to otitis media. Otol Neurotol. 2008; 29: 1056-1060

8. Levine OS, Farley M, Harrison LH, Lefkowitz L, McGeer A, et al. Risk factors for invasive pneumococcal disease in children: a population-based case-control study in North America. Pediatrics. 1999; 103: E28.

9. Pedersen CB, Gøtzsche H, Møller JO, Mortensen PB. The Danish Civil Registration System. A cohort of eight million persons. Dan. Med. Bull. 2006; 53: 441-449.

10. Harboe ZB, Thomsen RW, Riis A, Valentiner-Branth P, Christensen JJ, et al. Pneumococcal serotypes and mortality following invasive pneumococcal disease: a population-based cohort study. PLoS Med. 2009; 6: e1000081.

11. Konradsen HB, Kaltoft MS. Invasive pneumococcal infections in Denmark from 1995 to 1999: epidemiology, serotypes, and resistance. Clin. Diagn. Lab. Immunol. 2002; 9: 358-365.

12. Mackenzie GA, Leach AJ, Carapetis JR, Fisher J, Morris PS. Epidemiology of asopharyngeal carriage of respiratory bacterial pathogens in children and adults: cross-sectional surveys in a population with high rates of pneumococcal disease. BMC.Infect.Dis. 2010; 10: 304.

13. Kwambana BA, Barer MR, Bottomley C, Adegbola R A, Antonio $M$. Early acquisition and high nasopharyngeal co-colonization by Streptococcus pneumoniae and three respiratory pathogens amongst Gambian new-borns and infants. BMC Infect. Dis. 2011; 11: 175 .

14. Gray BM, Converse GM, Dillon HC. Epidemiologic studies of Streptococcus pneumoniae in infants: acquisition, carriage, and infection during the first 24 months of life. J. Infect. Dis. 1980; 142: 923-933.

15. Chapman KE, Wilson D, Gorton R. Invasive pneumococcal disease and socioeconomic deprivation: a population study from the North East of England. J. Public Health (Oxf). 2013; 35: 558569.

16. Regev-Yochay G, Dagan R, Raz M, Carmeli Y, Shainberg B, et al. Association between carriage of Streptococcus pneumoniae and Staphylococcus aureus in Children. JAMA 2004; 292: 716-720.

17. Feldman C, Anderson R. Review: Current and new generation pneumococcal vaccines. J. Infect. 2014.

18. Hausdorff WP, Bryant J, Paradiso PR, Siber GR. Which pneumococcal serogroups cause the most invasive disease: implications for conjugate vaccine formulation and use, part I. Clin. Infect. Dis. 2000; 30: 100-121.

19. Pneumococcal Disease: Prevention \& Treatment - IVAC - International Vaccine Access Center - Johns Hopkins Bloomberg School of Public Health.

20. van Aalst M, Lötsch F, Spijker R, van der Meer JTM, Langendam $\mathrm{MW}$, et al. Incidence of invasive pneumococcal disease in im- 
munocompromised patients: A systematic review and metaanalysis. Travel Med Infect Dis. 2018; 24: 89-100.

21. Singleton R, Wenger J, Klejka JA, Bulkow LR, Thompson A, et al. The 13-valent pneumococcal conjugate vaccine for invasive pneumococcal disease in Alaska native children: results of a clinical trial. Pediatr Infect Dis J. 2013; 32: 257-263.

22. Rückinger $\mathrm{S}$, von Kries $\mathrm{R}$, Siedler $\mathrm{A}$, van der Linden $\mathrm{M}$. Association of serotype of Streptococcus pneumoniae with the risk of severe and fatal outcome. Pediatr. Infect. Dis. J. 2003; 28: 118122.

23. Levy C, Varon E, Bingen E, Lécuyer A, Boucherat M, et al. Bacterial Meningitis Study Group. Pneumococcal meningitis in French children before and after the introduction of pneumococcal conjugate vaccine. The Pediatric infectious disease journal. 2011; 30: $168-70$.

24. Black SB, Shinefield HR, Hansen J, Elvin L, Laufer D, et al. Postlicensure evaluation of the effectiveness of seven valent pneumococcal conjugate vaccine. The Pediatric infectious disease journal. 2001 Dec 1; 20: 1105-1107.

25. Tomczyk S, Bennett NM, Stoecker C, Gierke R, Moore MR, et al. Use of 13-valent pneumococcal conjugate vaccine and 23-valent pneumococcal polysaccharide vaccine among adults aged $\geq 65$ years: Recommendations of the Advisory Committee on Immunization Practices (ACIP). MMWR. Morbidity and mortality weekly report. 2014; 63: 822

26. Moore CE, Paul J, Foster D, Mahar SA, Griffiths D, et al. Oxford Invasive Pneumococcal Surveillance Group: Reduction of invasive pneumococcal disease 3 years after the introduction of the 13valent conjugate vaccine in the Oxfordshire region of England. $J$ Infect Dis. 2014; 210: 1001-1011.

27. Harboe ZB, Valentiner-Branth P, Ingels H, Rasmussen JN, Andersen $\mathrm{PH}$, et al. 2013. Pediatric invasive pneumococcal disease caused by vaccine serotypes following the introduction of conjugate vaccination in Denmark. PLoS ONE. 2013 8: e51460.

28. Bryant KA, Block SL, Baker SA, Gruber WC, Scott DA; PCV13 Infant Study Group. Safety and immunogenicity of a 13-valent pneumococcal conjugate vaccine. Pediatrics. 2010; 125: 866875.

29. Grant LR, O'Brien SE, Burbidge P, Haston M, Zancolli M, et al. Comparative immunogenicity of 7 and 13 -valent pneumococcal conjugate vaccines and the development of functional antibodies to cross-reactive serotypes. PLoS One. 2013; 8: e74906.

30. Antachopoulos C, Tsolia MN, Tzanakaki G, Xirogianni A, Dedousi $\mathrm{O}$, et al. Parapneumonic pleural effusions caused by Streptococcus pneumoniae serotype 3 in children immunized with 13valent conjugated pneumococcal vaccine. Pediatr Infect Dis J. 2014; 33: 81-83.

31. Madhi F, Godot C, Bidet P, Bahuaud M, Epaud R, et al. Serotype 3 pneumococcal pleural empyema in an immunocompetent child after the 13-valent pneumococcal conjugate vaccine. Pediatr Infect Dis J. 2014; 33: 545-546.

32. Joye S, Gao A, Kayemba-Kay's S, Cotting J, Perez MH. Invasive pneumococcal infection despite the 7-valent conjugate vaccine. Clin Pract. 2013; 3: e11

33. Kayemba-Kay's S, Badran AM, Lagneaux C, Kovacs T, Heron A. Streptococcus pneumoniae serotype 19A meningitis in a wellvaccinated immunocompetent 13-month-old child: a case of PCV13 failure. Clin Case Rep. 2016; 4: 1023-1025.

34. Sütçü $M$, Aktürk $H$, Karagözlü $F$, Somer A, Gürler N, et al. Empyema due to streptococcus pneumoniae serotype $9 \mathrm{~V}$ in a child immunized with 13-valent conjugated pneumococcal vaccine.
Balkan Med J. 2017; 34: 74-77

35. Godot C, Levy C, Varon E, Picard C, Madhi F, et al. J Pediatr Infect Dis J 2015; 34: e260-e263.

36. Olarte L, Barson WJ, Barson RM, Lin PL, Romero JR, et al. Impact of the 13-Valent Pneumococcal Conjugate Vaccine on Pneumococcal Meningitis in US Children. Clinical Infectious Diseases. 2015; 61: 767-775.

37. Poolman J, Kriz P, Feron C, Di-Paolo E, Henckaerts I, et al. Pneumococcal serotype 3 otitis media, limited effect of polysaccharide conjugate immunization and strain characteristics. Vaccine. 2009; 27: 3213-3222.

38. Moorthy AN, Rai P, Jiao H, Wang S, Tan KB, et al. Capsules of virulent pneumococcal serotypes enhance the formation of neutrophil extracellular traps during the in vivo pathogenesis of pneumonia. Oncotarget. 2016; 7: 19327-19340.

39. Trück J, Thompson A, Morales-Aza B, Clutterbuck EA, Voysey M, et al. Memory $B$ cell response to a PCV-13 booster in 3.5-yearold children primed with either PCV-7 or PCV-13. Vaccine. 2017; 35: 2701-2708.

40. Heininger U, Bachtiar NS, Bahri P, Dana A, Dodoo A, et al. The concept of vaccination failure. Vaccine 2012; 30: 1265-1268.

41. Galanis I, Lindstrand A, Darenberg J, Browall S, Nannapaneni P, et al. Effects of PCV7 and PCV13 on invasive pneumococcal disease and carriage in Stockholm, Sweden. Eur Respir J 2016; 47: $1208-1218$

42. Moraga-Llop F, Garcia-Garcia JJ, Díaz-Conradi A, Ciruela P, Martínez-Osorio J, et al. Vaccine Failures in Patients Properly Vaccinated with 13-Valent Pneumococcal Conjugate Vaccine in CataIonia, a Region with Low Vaccination Coverage. Pediatr Infect Dis J. 2016 ; 35: 460-463.

43. Hernández S, Moraga-Llop F, Díaz A, de Sevilla MF, Ciruela P, et al. Failures of 13 -Valent Conjugated Pneumococcal Vaccine in Age-Appropriately Vaccinated Children 2-59 Months of Age, Spain. CDC Emerging Infectious Diseases 2020; 26.

44. Ladhani SN, Collins S, Djennad A, Sheppard CL, Borrow R, et al. A rapid increase in non-vaccine serotypes causing invasive pneumococcal disease in England and Wales, 2000-17: a prospective national observational cohort study. Lancet Infect Dis. 2018; 18: 441-451.

45. Zimmermann P, Perrett KP, Berbers G, Curtis N. Persistence of pneumococcal antibodies after primary immunization with a polysaccharide-protein conjugate vaccine. Arch Dis Child. 2019; 104: 680-684.

46. Jayasinghe S, Chiu C, Quinn H, Menzies R, Gilmour R, et al. Effectiveness of 7- and 13-valent pneumococcal conjugate vaccines in a schedule without a booster dose: a 10-year observational study. Clin Infect Dis 2018; 67: 367-374.

47. Blyth CC, Jayasinghe S, Chiu C, et al. Benefits of 13vPCV schedule switch from $3 p+0$ to $2 p+1$ in Australian children. Adelaide, Australia: Presented at 16th National Immunisation Conference, 2018.

48. Carville K. 2001-2016: serotypes and vaccine failures. Presented at 16th national immunization conference, Adelaide, Australia. Victoria, Australia: Invasive pneumococcal disease in children, 2018.

49. Andrews NJ, Waight PA, Burbidge $P$, Pearce $E$, Roalfe $L$, et al. Serotype-specific effectiveness and correlates of protection for the 13-valent pneumococcal conjugate vaccine: a postlicensure indirect cohort study. Lancet Infect Dis 2014; 14: 839-846. 